\section{Comparation of the new rebound tonometer IOPen and the Goldmann tonometer, and their relationship to corneal properties}

J Moreno-Montañés', I Gosende', J Caire' and M García-Granero²
1Department of

Ophthalmology, Clínica Universidad de Navarra, Pamplona, Spain

${ }^{2}$ Department of Genetics, Unidad de Estadística, Universidad de Navarra, Pamplona, Spain

Correspondence: J MorenoMontañés, Department of Ophthalmology, Clínica Universidad de Navarra, Avda. Pío XII 36, Pamplona 31008, Spain

Tel: + 948 296331;

Fax: + 948296500

E-mail: jmoreno@

unav.es

Received: 10 January 2010 Accepted in revised form: 19 August 2010 Published online: 1 October 2010

\begin{abstract}
Purpose To compare the intraocular pressures (IOPs) obtained with the IOPen rebound tonometer, Goldmann applanation tonometer (GAT) and the ocular response analyzer (ORA) and investigate the effects of corneal biomechanical properties on IOPen measurements.

Methods A total of 198 normal eyes were included in this cross-sectional and randomized study. Three measurements were taken using IOPen. Agreement between tonometers was calculated using the Bland and Altman limits of agreement (LoA) analysis.

Results The median IOPen IOP was $3 \mathrm{~mm} \mathrm{Hg}$ below the GAT $(P<0.001), 3 \mathrm{~mm} \mathrm{Hg}$ below the ORA IOP similar to Goldmann (IOPg), and $3 \mathrm{~mm} \mathrm{Hg}$ below the ORA IOP corrected using corneal parameters (IOPcc) $(P<0.01)$. The LoA width between the IOPen and GAT IOPs varied between 13.92 (mean IOPen IOP) and $15.99 \mathrm{~mm} \mathrm{Hg}$ (third IOPen measurement). The central corneal thickness (CCT) was unrelated to IOPen measurements $(P>0.05)$. Corneal hysteresis $(\mathrm{CH})$ and corneal rigidity factor (CRF) were correlated with IOPen and GAT.

Conclusions IOPen underestimated the IOP compared with GAT and ORA. The effect of measurement quality or measurement order on IOPen was low. CCT did not affect the IOPen, but the $\mathrm{CH}$ and CRF did. The LoA width between the IOPen and GAT IOPs was higher than between the ORA IOPg or ORA IOPcc and GAT IOPs. Eye (2011) 25, 50-56; doi:10.1038/eye.2010.137; published online 1 October 2010
\end{abstract}

Keywords: corneal properties; goldmann tonometer; ocular response analyzer; rebound tonometer

The rebound tonometer (RT), which has become one of the most promising devices for measuring intraocular pressure (IOP), has a stainless steel probe $50 \mathrm{~mm}$ long and $1.4 / 1.0 \mathrm{~mm}$ in diameter that is repelled horizontally by a coaxial twomagnet system and hits the cornea at a distance of $4-8 \mathrm{~mm}$. The rebounding probe induces voltage in the solenoid that is converted to a digital signal. RTs make it easy to measure the IOP in children and uncooperative patients (elderly subjects with poor cooperation or dementia), and no corneal anesthesia is required. ${ }^{1,2}$ The round tip minimizes the risk of corneal injury from the probe; the tip is disposable, which decreases the risk of corneal infections. Finally, the RT tip has a plastic cover $1-\mathrm{mm}$ in diameter that facilitates IOP measurement in patients with corneal irregularities or after a penetrating keratoplasty. ${ }^{1}$

The ICare (Tiolat, Helsinki, Finland), the first RT to become available, obtained official approval (CE mark) and more than 15000 ICare are used in over 50 countries. Recently, a new RT named IOPen (Medicel AG, Wolfhalden, Switzerland) has become available. This new RT has advantages in relation to ICare: it can be used at the slit lamp (Figure 1); and the IOP is measured perpendicular to the center of the cornea to avoid incorrect rebound. When the RT is perpendicular to the cornea, a green light is reflected to the cornea; if the RT is not well situated, a red light is reflected in the cornea and the IOP cannot be measured (Figure 2). Finally, according to the manufacturer, the IOP 


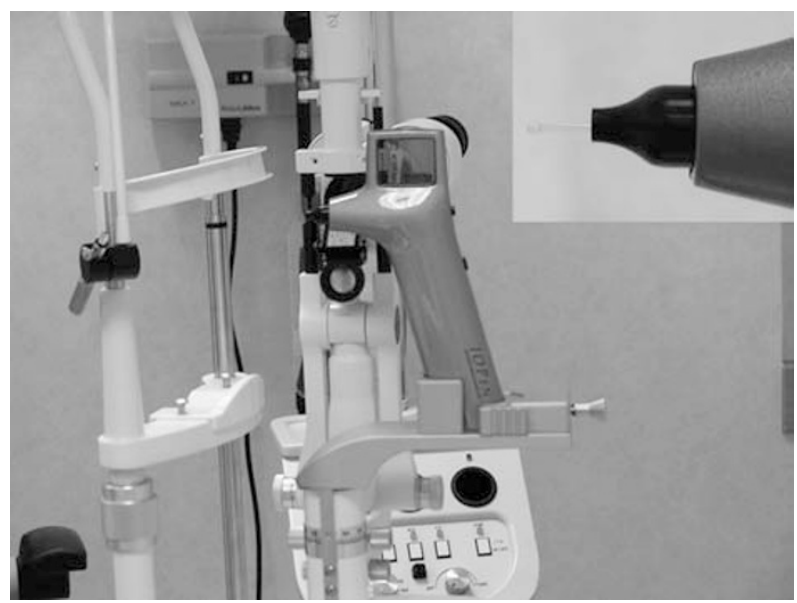

Figure 1 Use of the IOPen tonometer at the slit lamp. The tip characteristics are shown in the box.

obtained using the IOPen is unaffected by the central corneal thickness (CCT).

The goal of the current study was to determine a correlation between the IOP values measured by IOPen and the Goldmann applanation tonometer (GAT), and the IOP values measured using the ocular response analyzer (ORA, Reichert, Depew, NY, USA) in normal eyes. We also studied the effect of the CCT and biomechanical properties obtained using the ORA on IOP measurements obtained using the IOPen RT.

\section{Materials and methods}

We conducted a randomized, cross-sectional study that compared the IOP measurements obtained using GAT, IOPen, and the ORA. Subjects were recruited from among the hospital staff, relatives of patients, and subjects without ocular diseases referred for a routine visual acuity examination. The institutional review board/ethics committee approved the study protocol. Each subject provided informed consent according to the tenets of the Declaration of Helsinki. All participants were Caucasian and underwent a complete ophthalmic examination, including recording of medical and family histories, visual acuity measurement, GAT, and anterior and posterior segment slit-lamp examinations. Normal eyes were defined as those with no previous history of ocular trauma or corneal pathologies or previous ocular surgery. Patients with contact lens, corneal pathologies (defined as eyes with penetrating keratoplasty, corneal edema, keratoconus, corneal scarring, microcorneas or megalocorneas, or band keratopathy) and cornea guttata were excluded. All subjects had a spherical equivalent of 5.0 diopters or less and astigmatism of 3.0 diopters or less.

The same examiner (IG) obtained all IOP measurements using the same tonometers in a random

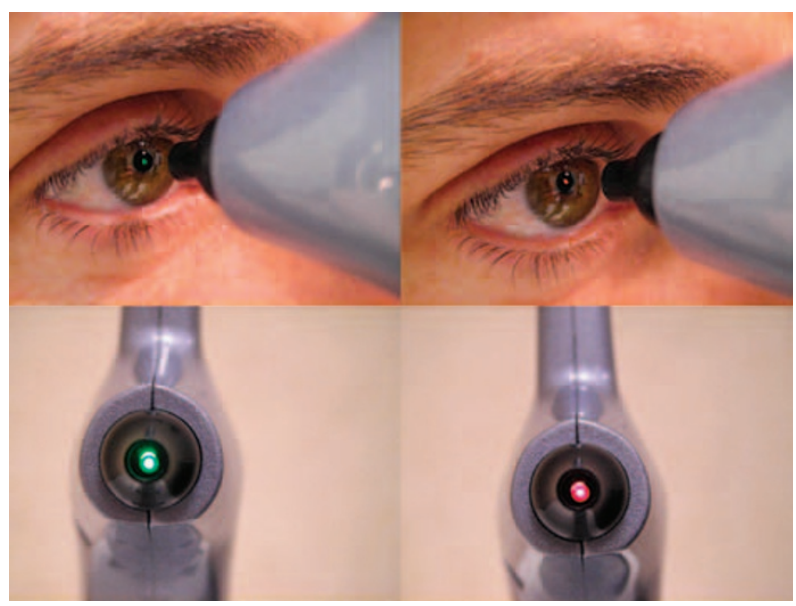

Figure 2 The corneal color reflex indicates that the IOPen is perpendicular to the corneal centre (indicated by green in the left figure) or it is not perpendicular to the corneal centre (indicated by red in the right figure).

order. The first 20 measurements obtained using the IOPen were rejected and considered as the learning curve. Corneal anesthesia (one drop of $0.5 \%$ proparacaine) was instilled before IOP measurements in all cases, because some subjects had difficulty in cooperating with the IOPen without anesthetic drops during the learning curve. Three IOP measurements were obtained using the IOPen (each measurement was the mean of six readings and an average value was generated automatically), and the mean of these three measurements was calculated. The measurement quality was rated on a scale ranging from 0 (better quality) to 5 (worse quality). Only good-quality (0-3) IOPen measurements were recorded; if the score was 4 or 5 , a new measurement was obtained. The best quality IOPen measurement was also analyzed. The same examiner used the ORA and four data points were recorded: IOP similar to Goldmann (IOPg), IOP corrected using corneal parameters (IOPcc), corneal hysteresis $(\mathrm{CH})$, and corneal rigidity factor (CRF). The CCT was measured using ORA pachymetry. At each CCT measurement, the probe was aligned perpendicular to the center of the cornea.

According to international requirements for standardizing ocular tonometers (International Organization for Standardization 8612), a calculation was made of the percentage of eyes that had an IOP that differed from GAT by more than $\pm 5 \mathrm{~mm} \mathrm{Hg}$ in each of the IOPen measurements and in each of the ranges: $7-16 \mathrm{~mm} \mathrm{Hg}$, and $>16-<23 \mathrm{~mm} \mathrm{Hg}$.

\section{Statistical analysis}

The Shapiro-Wilk test was used to assess the normality of the quantitative variables. Normally distributed 
variables ( $\mathrm{CH}, \mathrm{CCT}$, and $\mathrm{CRF}$ ) were summarized using mean $\pm S D$, and non-normally distributed variables (IOP) were summarized using the median and interquartile range. To compare quantitative variables between independent groups, the Mann-Whitney $U$ - and Wilcoxon tests were used for non-normally distributed variables. Association between IOPen and CCT were calculated by lineal regression. The associations between the IOP obtained with IOPen and CCT, $\mathrm{CH}$, and CRF were studied using a multiple regression model, with IOPen as the dependent variable and $\mathrm{CCT}, \mathrm{CH}$, and CRF as independent variables. To assess the validity of the model, the normality of the residuals was verified by the means of the Shapiro-Wilk test. Similarly, a multiple regression model was made between GAT and the same corneal parameters. The agreement of the IOP values among the tonometers (quantitative data) was evaluated using the Bland-Altman method. ${ }^{3}$ The $95 \%$ limits of agreement (LoA) were also calculated. Data were assessed using the SPSS program (version 15.0.1, SPSS Inc, Chicago, IL, USA), and MedCalc (version 9.2, MedCalc Software, Mariakerke, Belgium). The level of signicance for each contrast was $P<0.05$.

\section{Results}

A total of 198 eyes of 198 subjects ( 57 men, 141 women; median age, 50 years; interquartile range, $25-71$ years) were included in this study. One eye of each subject was chosen randomly (100 right eyes, 98 left eyes). The mean \pm SD spherical equivalent was $0 \pm 1.75$ (median, 0 ).

A total of 112 eyes were emmetropic.

The IOP medians using the tonometers and the parameters evaluated are shown in Table 1 . No differences were found between the GAT IOP and the ORA IOPg $(P=0.23)$ or between GAT IOP and ORA IOPcc $(P=0.06)$. There were significant differences between each IOPen value, the mean IOPen, and the best-quality IOPen compared with GAT $(P<0.001)$ and the ORA IOPs $(P<0.01)$. In 37 cases $(18.7 \%)$, the mean IOPen IOP was higher than the GAT IOP. The IOP values from both tonometers were the same in only two eyes. In 159 cases $(80.3 \%)$, the GAT IOP was higher than the mean IOPen IOP. In 36 cases (18.2\%), the difference between the mean IOPen and GAT IOPs was $1 \mathrm{~mm} \mathrm{Hg}$ or less, and in 61 eyes $(30.8 \%)$ it was $2 \mathrm{~mm} \mathrm{Hg}$ or less; in 99 eyes $(50 \%)$, it was $3 \mathrm{~mm} \mathrm{Hg}$ or less. However, if the GAT IOP exceeded $20 \mathrm{~mm} \mathrm{Hg}$ (seven cases), the median difference between the GAT and mean IOPen IOPs was $13 \mathrm{~mm} \mathrm{Hg}$ (interquartile range, 12-15.3). The percentages of eyes with differences between IOPen and GAT IOP values greater than $\pm 5 \mathrm{~mm} \mathrm{Hg}$ were $32 \%$ for the first IOPen measurement, $34.3 \%$ for the second IOPen value, $30.3 \%$ for the third IOPen measurement, $28.2 \%$ for the mean
Table 1 Summary of the IOP measurements ( $\mathrm{mm} \mathrm{Hg}$ ) using the three tonometers and the corneal parameters

\begin{tabular}{lccc}
\hline Data & Mean $\pm S D$ & $\begin{array}{c}\text { Median } \\
(25-75 \% \text { IQR })\end{array}$ & P-value $^{\mathrm{a}}$ \\
\hline GAT IOP & $14.6 \pm 2.9$ & $14(13-16)$ & \\
IOPen IOP 1 & $11.3 \pm 3.4$ & $11.5(9-13.5)$ & 0.001 \\
IOPen IOP 2 & $11.2 \pm 3.6$ & $10.7(9-13)$ & 0.001 \\
IOPen IOP 3 & $11.6 \pm 3.5$ & $11(9-14)$ & 0.001 \\
Mean IOPen IOP & $11.4 \pm 2.9$ & $11.33(9.2-13.2)$ & 0.001 \\
Best quality IOPen IOP & $11.2 \pm 3.4$ & $11(8.5-13)$ & 0.001 \\
IOPg (ORA) & $14.4 \pm 4$ & $13.9(11.6-16.6)$ & 0.23 \\
IOPcc (ORA) & $15 \pm 4$ & $14.8(11.9-17.4)$ & 0.06 \\
Pachymetry (microns) & $551.3 \pm 35.2$ & $555(523-577)$ & \\
CH (mm Hg) & $10.5 \pm 1.7$ & $10.4(9.4-11.6)$ & \\
CRF (mm Hg) & $10.2 \pm 1.9$ & $10.2(8.8-11.4)$ & \\
\hline
\end{tabular}

Abbreviations: $\mathrm{CH}$, corneal hysteresis; $\mathrm{CRF}$, corneal rigidity factor; $\mathrm{GAT}$, Goldmann applanation tonometer; IOP, intraocular pressure; IQR, interquartile range; ORA, ocular response analyzer.

${ }^{a}$ Compared to IOP Goldmann.

IOPen value, and $34.3 \%$ for the best-quality IOPen value. The percentage for the difference between mean IOPen and GAT IOP values greater than $\pm 5 \mathrm{~mm} \mathrm{Hg}$ was $21.25 \%$ for the range $7-16 \mathrm{~mm} \mathrm{Hg}$ and $62 \%$ for the range $>16-<23 \mathrm{~mm} \mathrm{Hg}$. The group with an IOP $\geqslant 23 \mathrm{~mm} \mathrm{Hg}$ did not include a sufficiently large number of eyes for this assessment.

The mean scores of the IOPen quality were $1.21 \pm 0.93$, $1.10 \pm 0.90$ and $1.11 \pm 0.89$ for the first, second, and third IOPen measurements, respectively. No differences in quality were found between the three IOPen measurements $(P=0.21)$. The mean score of the best-quality IOPen measurement was $0.52 \pm 0.63$.

The IOP medians distributed according to gender and age (two groups) are shown in Table 2. The IOP values comparison between men and women of the IOPen was significant statistically for the first, second IOPen measurements and for the mean IOP IOPen $(P<0.05)$. However, no difference was found in IOP GAT $(P=0.98)$, ORA IOPg $(P=0.91)$, and ORA IOPcc $(P=0.41)$. The IOP values comparison according to two groups of age $(<50$ and $\geqslant 50$ years) showed statistical differences between IOPen and ORA $(P<0.05)$, but not in the IOP GAT $(P=0.11)$.

Figure 3 and Table 3 show the Bland-Altman analysis and $95 \%$ LoAs. The $95 \%$ LoAs width using the mean IOPen measurement was lower than the 95\% LoAs in the bestquality IOPen measurement. However, the mean difference and 95\% LoAs between the ORA and GAT IOPs was lower than all IOP measurements using IOPen and GAT.

The mean IOPen measurement, each measurement, and the best quality IOPen measurement were unrelated to the CCT $(P>0.05)$ (Figure 4). However, GAT was correlated with the CCT $\left(R^{2}, 0.08 ; P=0.001\right)$. The ORA IOPg was correlated with the CCT $\left(R^{2}, 0.11 ; P=0.001\right)$; 
Table 2 Median (25-75\% IQR) of the IOP measurements ( $\mathrm{mm} \mathrm{Hg}$ ) using the three tonometers distributed according to gender and age $(<50$ and \pm 50 years old $)$

\begin{tabular}{|c|c|c|c|c|}
\hline & \multicolumn{2}{|c|}{ Gender } & \multicolumn{2}{|c|}{ Age distribution } \\
\hline & Men & Women & $<50$ & \pm 50 \\
\hline IOP Goldmann & $14(12-16)$ & $14(13-16)$ & $14(13-16)$ & $15(13-16.5)$ \\
\hline IOP IOPen $1^{\ddagger \#}$ & $12(10.5-15)$ & $11(8.5-13)$ & $10.5(7.8-12.5)$ & $12(10.5-14.5)$ \\
\hline IOP IOPen $2^{\ddagger \#}$ & $12(9.5-14.2)$ & $10.5(8.5-12.8)$ & $10(8.5-12.5)$ & $12(10-15)$ \\
\hline IOP IOPen $3^{\#}$ & $12(10-14)$ & $11(8.5-14)$ & $10(8.5-12.5)$ & $12.5(10.5-14.5)$ \\
\hline Mean IOP IOPen & $12.2(10.3-13.8)$ & $11(8.5-13)$ & $10.3(8.5-12.1)$ & $12.6(10.6-14.2)$ \\
\hline Best IOPen quality ${ }^{\#}$ & $11.5(10-14.3)$ & $10.5(8.3-13)$ & $10(7.5-12)$ & $12(10-15)$ \\
\hline IOPg $(\mathrm{ORA})^{\#}$ & $14(11.5-16.6)$ & $13.9(11.6-16.5)$ & $13(11.1-15.7)$ & $15.4(12.2-18)$ \\
\hline IOPcc (ORA) $)^{\#}$ & $15.6(13-18.2)$ & $15(11.5-16.7)$ & $13.5(10.8-15.5)$ & $16.3(13.6-19)$ \\
\hline
\end{tabular}

Abbreviations: IOP, intraocular pressure; IQR, interquartile range; ORA, ocular response analyzer.

${ }^{\ddagger} P<0.05$ Mann-Withney test (gender comparison).

${ }^{\#} P<0.05$ Mann-Withney test (age groups comparison).

and the IOPcc was not correlated with the CCT $\left(R^{2}, 0.007\right.$; $P=0.23$ ). Multiple regression analysis showed significant associations between the mean IOPen measurement and the $\mathrm{CH}$ (slope, $-1.22 ; P<0.001$ ) and the $\mathrm{CRF}$ (slope, 1.91 ; $P<0.001$ ) (model fit $R_{\mathrm{adj}}^{2}$ 0.219); the association between the mean IOPen IOP and the CCT was not significant (slope, $-8^{*} 10^{-3} ; P=0.16$ ). Similarly, a significant association was seen between the GAT and the $\mathrm{CH}$ (slope, 1.57; $P<0.001$ ), and CRF (slope, $-1.98 ; P<0.001$ ) (model fit $R_{\text {adj }}^{2}$ 0.698 ); the association between GAT and the CCT was not significant (slope, $-5^{*} 10^{-4} ; P=0.86$ ). The difference between IOPen values and IOP GAT were not related to spherical equivalent or $\mathrm{CH}$ or $\mathrm{CRF}(P<0.05)$.

\section{Discussion}

The GAT is the most widely used device for measuring IOP, and it is considered the gold standard tonometer. However, a correction algorithm for the GAT may not be accurate for estimating the IOP in individual subjects. Thus, corneal parameters, especially the $\mathrm{CCT}$, affect the accuracy of this tonometer. ${ }^{4}$ Several corneal conditions, such as high astigmatism, penetrating keratoplasty, irregularities in corneal curvature, Fuchs' dystrophy associated with laser in situ keratomileusis, or altered ocular surface hydration, could affect IOP measurements, and GAT is not useful in all cases with corneal pathologies. ${ }^{1,4,5}$ The RT was developed from animal studies, ${ }^{6}$ and now has become available for use in humans. The RT also is used by general practitioners in glaucoma screening programs, optometrists, and occupational healthcare personnel because it performs well even for inexperienced practitioners. ${ }^{7}$ The 1-mmdiameter disposable tip of the RT facilitates IOP measurements and avoids corneal infections and could be used in corneas with keratoconus, ulcers, edema, or graft rejection after penetrating keratoplasty. ${ }^{1}$ Similar to GAT, the IOPen is the first RT that can be used at the slit lamp. In the current study, we compared this RT with the GAT. To our knowledge no previous reports have been published about the IOPen and their relationship to biomechanical corneal properties.

The studies that compared the RT and GAT reported that the ICare RT overestimated the mean IOP between 0.5 and $2 \mathrm{~mm} \mathrm{Hg}$ compared with GAT. ${ }^{1,8-11}$ In the current study using IOPen, the median IOP was $3 \mathrm{~mm} \mathrm{Hg}$ lower than the GAT or ORA IOPs. Other studies of the ICare reported that $63.2 \%$ of cases had IOP values that differed from the IOP values obtained with GAT by $3 \mathrm{~mm} \mathrm{Hg}$ or less, a limit considered clinically relevant. ${ }^{10}$ In our previous study of normal corneas, we found that the difference between GAT and I ICare IOPs was $3 \mathrm{~mm} \mathrm{Hg}$ or less in $87.5 \%$ of eyes. ${ }^{1}$ Other authors obtained a similar result. ${ }^{11}$ In the current study, a difference between IOPen and GAT of $3 \mathrm{~mm} \mathrm{Hg}$ or less was found in 99 eyes (50\%), which is lower than in previous reports about ICare. ${ }^{1,10,11}$ Finally, in the current study the width of the 95\% LoA in the Bland-Altman plot of differences between GAT and IOPen IOPs was $13.92 \mathrm{~mm} \mathrm{Hg}$ for the mean IOPen value and $15.99 \mathrm{~mm} \mathrm{Hg}$ for the best quality IOPen values. In our previous study, the width of the 95\% LoA between GAT and ICare was $10.96 \mathrm{~mm} \mathrm{Hg}^{1}$ Other authors reported a width of the 95\% LoA between GAT and ICare between $8.9^{11}$ and $11 \mathrm{~mm} \mathrm{Hg}$. ${ }^{9,10}$ Those results suggested that the ICare obtains better results than the IOPen compared with GAT. In addition, the percentage of eyes with differences between IOPen and GAT greater than $\pm 5 \mathrm{~mm} \mathrm{Hg}$ was about $30 \%$ in all IOPen measurements. According to the IOP ranges established by the International Organization for Standardization 8612, differences between IOPen and GAT measurements exceeding $\pm 5 \mathrm{~mm} \mathrm{Hg}$ were in more than the accepted level of 5\% of subjects. The IOPen does not meet the International Organization for Standardization 8612 requirements.

The disagreement between IOPen and ICare IOPs could result from patient cooperation, systematic errors of tonometry, changes in the modulus of the corneal 
elasticity, and tear film characteristics. ${ }^{4}$ One of the most important is the effect of the corneal properties on IOP measurements. Thus, the IOPen obtains the measurement in the centre of the cornea and the rebound is perpendicular to the corneal surface. The ICare does not need to situate the IOP in the corneal centre. Some authors suggested a difference of $0.6 \mathrm{~mm} \mathrm{Hg}$ when using the ICare to obtain IOP values in the centre or corneal
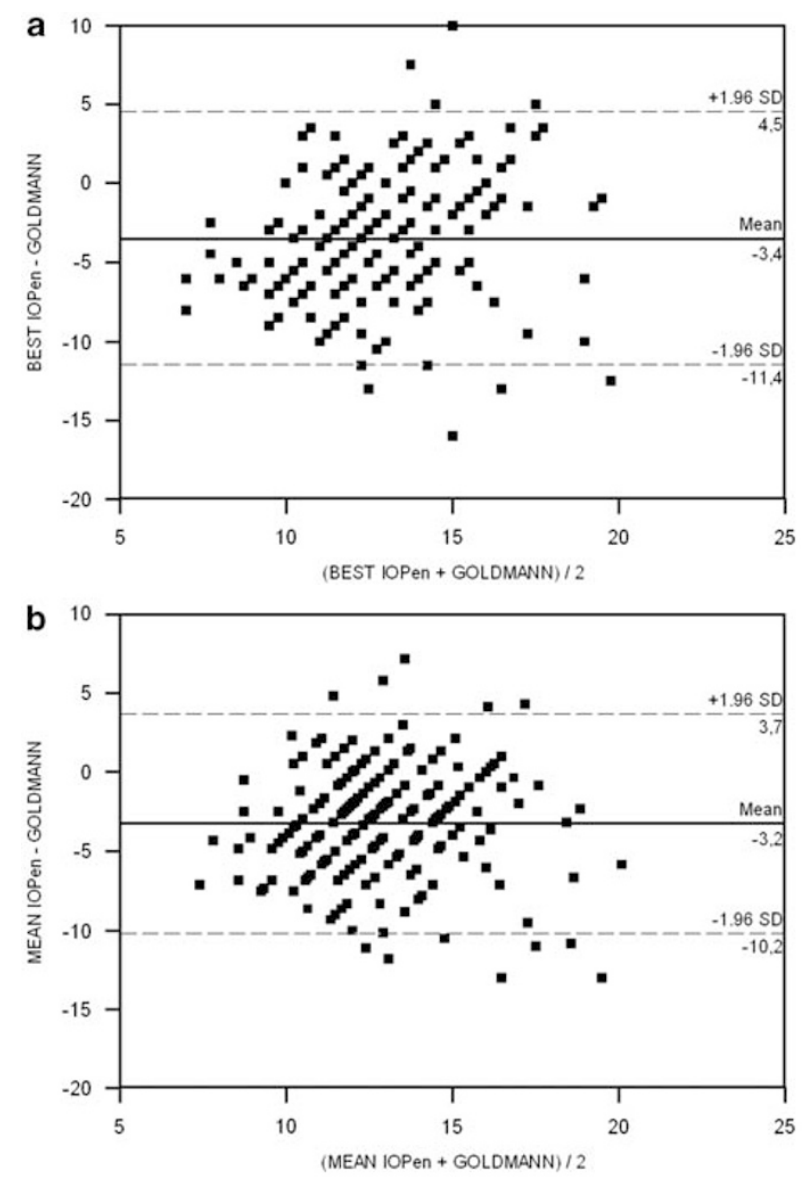

Figure 3 Top (a), the Bland-Altman plot of the means against the differences between the best IOPen and GAT IOPs. Bottom (b), the Bland-Altman plot of the means against the differences between the mean IOPen measurements and GAT IOPs. periphery. ${ }^{10}$ Another difference in the current study from the ICare was that the IOPen was unaffected by the CCT. Other authors found that ICare IOPs was modified by CCT variations. ${ }^{9,12}$ However, the IOPen was affected by the $\mathrm{CH}$ and CRF. Similarly, Chui et al found that the $\mathrm{CH}$ and CRF modified the IOP results when using ICare. ${ }^{10}$ Other tonometers, such as the TonoPen (Mentor,

Norwell, MA, USA) also have a relationship with the $\mathrm{CH}$ and CRF. ${ }^{13}$ Mollan et al suggested that corneal parameters, such as the $\mathrm{CH}$ and CRT may be more important than the CCT in causing inaccuracies in different tonometers. ${ }^{13}$

In Table 2 the IOP comparison among tonometers according to gender and age is shown. The mean IOPen measurements varied in men and women. However, this difference was not found in IOP GAT, or ORA IOPg, or ORA IOPCC. Previous studies have found that the corneal properties are not related to gender. ${ }^{14}$ In relation to age groups, IOP values increased with age using IOPen and ORA, but not using GAT. In our previous report, we found the $\mathrm{CH}$ and $\mathrm{CRF}$ decreased with increasing age in healthy adults. Also the ORA IOPcc increased with age. ${ }^{15}$ The IOP results obtained using IOPen suggest that this tonometer is influenced by some corneal properties and

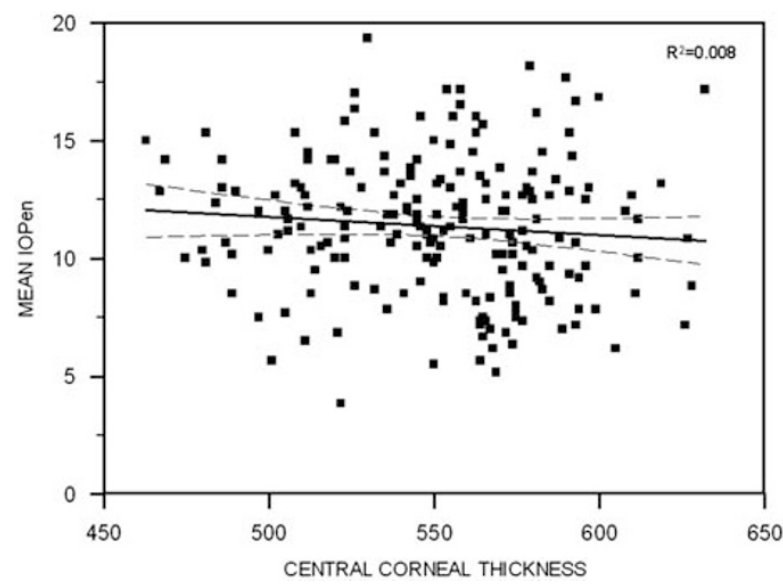

Figure 4 The association between the mean IOPen measurement and CCT $\left(R^{2}, 0.008 ; P=0.21\right)$.

Table 3 Bland-Altman analysis of IOP measurements using different tonometers (mean difference \pm SD and 95\% LoAs)

\begin{tabular}{lcccc}
\hline & Mean difference \pm SD (GAT IOP-IOP tonometers) & \multicolumn{2}{c}{$95 \%$ LoA } \\
\cline { 3 - 5 } & & Lower LoA & Upper LoA & Width of LoA \\
\hline IOP IOPen 1 & $3.3 \pm 4$ & -4.6 & 11.2 & 15.8 \\
IOP IOPen 2 & $3.4 \pm 4$ & -4.4 & 11.2 & 15.6 \\
IOP IOPen 3 & $3 \pm 4.1$ & -5 & 11 & 16 \\
Mean IOP IOPen & $3.2 \pm 3.6$ & -3.7 & 10.2 & 13.9 \\
Best IOPen quality & $3.4 \pm 4.1$ & -4.5 & 11.4 & 15.9 \\
IOPg (ORA) & $0.2 \pm 2.3$ & -4.2 & 4.6 & 8.8 \\
IOPcc (ORA) & $-0.4 \pm 2.8$ & -5.8 & 5 & 10.8 \\
\hline
\end{tabular}

Abbreviations: IOP, intraocular pressure; LoA, limits of agreement; GAT, Goldmann applanation tonometer; ORA, ocular response analyzer. 
there is a systematic error that underestimate the IOP compared with GAT in all groups of age or gender. This underestimation is probably not related to rebound method to take the IOP as the tonometer itself.

The current study had some limitations. First, most subjects were women, because there were a high number of nurses in the sample. However, as we have mentioned the gender did not affect the corneal properties. ${ }^{14}$ Also, the age distribution was wide because previous reports found that age may modify the corneal properties in normal eyes. ${ }^{15,16}$ Second, no patients with glaucoma were included. Only seven eyes had an IOP exceeding 20 using GAT and the median difference between the GAT and IOPen IOPs was $13 \mathrm{~mm} \mathrm{Hg}$ (cases with high CCT values); thus, the difference between the GAT and IOPen IOPs in eyes with high or low IOP ranges or in cases with thick CCT is not well known. However, the goal in the current study was only to determine the IOPen values in normal eyes. Third, all IOP measurements using the three tonometers were taken after instillation of anesthetic drops, which may change the corneal biomechanical parameters. ${ }^{17}$ However, in the IOPen learning curve, we found that without corneal anesthesia, the IOPen was uncomfortable for most subjects. Also the drops were instilled before the IOP measurements and the influence for the corneal properties modifications is similar for all tonometers. Despite these limitations, the current study was a prospective and randomized evaluation to determine the IOPen characteristics as a RT in normal eyes.

In conclusion, the median IOP measured using the IOPen RT was $3 \mathrm{~mm} \mathrm{Hg}$ lower than the GAT and the IOPg and IOPcc from the ORA tonometer. The effect of the measurement quality on the IOPen was low. The BlandAltman plot showed that the 95\% LoA measured using the IOPen was higher than the IOP measured using the ORA. A lower 95\% LoA was obtained with the mean IOPen values. The IOPen was unaffected by the CCT, but was correlated with $\mathrm{CH}$ and CFR. Further studies are needed of IOP measurements using the IOPen in glaucomatous eyes or eyes with corneal pathologies.

None of the authors has a financial, commercial, or proprietary interest in any of the instruments mentioned in this report.

\section{Summary}

What was known before

- The ICare is the first rebound tonometer with a good agreement with Goldmann tonometer.

What this study adds

- This study adds the clinical experience with a new rebound tonometer.

\section{Conflict of interest}

The authors declare no conflict of interest.

\section{Acknowledgements}

Supported in part by RETICS RD 07/0062 (Oftalmología), Instituto de Salud Carlos III, Madrid, Spain.

\section{References}

1 Moreno-Montañés J, García N, Fernández-Hortelano A, García-Layana A. Rebound tonometer compared with Goldmann tonometer in normal and pathologic corneas. Cornea 2007; 26: 427-430.

2 Sahin A, Basmak H, Niyaz L, Yildirim N. Reproducibility and tolerability of the ICare rebound tonometer in school children. J Glaucoma 2007; 16: 185-188.

3 Bland JM, Altman DG. Statistical methods for assessing agreement between two methods of clinical measurement Lancet 1986; 1: 307-310.

4 Chihara E. Assessment of true intraocular pressure: the gap between theory and practical data. Surv Ophthalmol 2008; 53: 203-218.

5 Moreno-Montañés J, Olmo N, Zarranz-Ventura J, HerasMulero H. Dynamic contour tonometry in eyes after penetrating keratoplasty. Cornea 2009; 28: 836-837.

6 Goldblum D, Kontiola AI, Mittag T, Chen B, Danias J. Noninvasive determination of intraocular pressure in the rat eye. Comparison of an electronic tonometer (TonoPen), and a rebound (impact probe) tonometer. Graefes Arch Clin Exp Ophthalmol 2002; 240: 942-946.

7 Abraham LM, Epasinghe NC, Selva D, Casson R. Comparison of the ICare rebound tonometer with the Goldmann applanation tonometer by experienced and inexperienced tonometrists. Eye 2008; 22: 503-506.

8 Davies LN, Bartlett H, Mallen EA, Wolffsohn JS. Clinical evaluation of rebound tonometer. Acta Ophthalmol Scand 2006; 84: 206-209.

9 Martinez-de-la-Casa JM, Garcia-Feijoo J, Castillo A, Garcia-Sanchez J. Reproducibility and clinical evaluation of rebound tonometry. Invest Ophthalmol Vis Sci 2005; 46: 4578-4580.

10 Chui WS, Lam A, Chen D, Chiu R. The influence of corneal properties on rebound tonometry. Ophthalmology 2008; 115: 80-84.

11 Fernandes P, Díaz-Rey JA, Queirós A, Gonzalez-Meijome JM, Jorge J. Comparison of the ICARE rebound tonometer with the Goldmann tonometer in a normal population. Ophthalmic Physiol Opt 2005; 25: 436-440.

12 Brusini P, Salvetat ML, Zeppieri M, Tosoni C, Parisi L. Comparison of ICare tonometer with Goldmann applanation tonometer in glaucoma patients. J Glaucoma 2006; 15: 213-217.

13 Mollan SP, Wolffsohn JS, Nessim M, Laiquzzaman M, Sivakumar S, Hartley S et al. Accuracy of Goldmann, ocular response analyser, Pascal and TonoPen XL tonometry in keratoconic and normal eyes. Br J Ophthalmol 2008; 92: 1661-1665.

14 Chang PY, Chang SW, Wang JY. Assessment of corneal biomechanical properties and intraocular pressure 
with Ocular Response Analyzer in childhood myopia. Br J Ophthalmol 2010; 94: 877-881.

15 Moreno-Montañés J, Maldonado MJ, García N, Mendiluce L, García-Gómez PJ, Seguí-Gómez M. Reproducibility and clinical relevance of the ocular response analyzer in nonoperated eyes: corneal biomechanical and tonometric implications. Invest Ophthalmol Vis Sci 2008; 49: 968-974.
16 Kotecha A, Elsheikh A, Roberts CR, Zhu H, Garway-Heath DF. Corneal thickness-and age-related biomechanical properties of the cornea measured with the ocular response analyzer. Invest Ophthalmol Vis Sci 2006; 47: 5337-5347.

17 Dayanir V, Sakarya R, Ozcura F, Kir E, Aktunç T, Ozkan BS et al. Effect of corneal drying on central thickness. J Glaucoma 2004; 13: 6-8. 